\title{
Current and Future Approaches to Stem Cell Treatment of Type I Diabetes
}

\author{
Michael Yacoub, ${ }^{*, a}$ Matthew Webert, ${ }^{*, a}$ Michael Lindberg, ${ }^{*, a}$ Marissa Viola*,a \\ ${ }^{a}$ Georgetown University School of Medicine, 3900 Reservoir Rd NW, Washington, DC 20007 \\ "authors contributed equally to this work
}

\begin{abstract}
This review analyzes the efficacy of current and previous stem cell therapies for type 1 diabetes (T1D) and suggests the most viable stem cell-based treatments for the future. T1D is an autoimmune disease in which the $\beta$ cells of the pancreas are destroyed. Currently, the only insulin replacement therapy options for T1D include insulin injection (which requires daily dosing) or organ transplantation (which has donor limitations and requires immunosuppression). Both options decrease a patient's quality of life. After reviewing completed clinical trials using stem cell therapy, we found that the combination therapy of adipose-derived stem cells and hematopoietic stem cells offers the greatest benefit for generating insulin-producing cells and modulating the autoreactivity of the immune system. However, this therapy is limited in scope and requires immunosuppression. The most promising potential treatment is encapsulation (the enclosure of stem cell-derived $\beta$ cells to prevent interactions with the immune system) because it can restore insulin production and does not require immunosuppression. Although encapsulation is in the early stages of development and has no completed clinical trials, it offers an ideal, long-term therapy option without compromising patients' quality of life and without requiring immunosuppression.
\end{abstract}

\section{Introduction}

Three million Americans are affected by type 1 diabetes (T1D), ${ }^{1}$ a disease process that is characterized by autoimmune destruction of glucose-sensing, insulin-producing $\beta$ cells located in the islets of the pancreas. $\beta$-cell depletion dysregulates metabolism and blood glucose levels due to a decrease in insulin production. The current standard of care is insulin replacement therapy with exogenous insulin via injection from a pen or pump. These treatment regimens can be burdensome, dangerous, and cumbersome owing to constant monitoring of blood sugar, self-dosing errors, and the need to carry extracorporeal devices and supplies, respectively. Transplantation, either whole islets or full pancreas, is an alternative to exogenous insulin injections. However, to mitigate risk of rejection, transplantation requires long-term immunosuppression and possibly repeated procedures. ${ }^{2}$ When determining whether allogeneic transplantation is necessary, health care professionals are forced to decide whether persistent immunosuppression would offer a better quality of life for a patient with T1D compared with life-long glucose monitoring paired with exogenous insulin. Transplantation is only recommended if glycemic control cannot be achieved via insulin injection. ${ }^{2}$ Along with immunosuppression, the key issue with transplantation is that the demand is greater than the supply. That is, the rate of available donor pancreases is severely outpaced by the incidence of T1D. ${ }^{3}$ Investigators have looked to stem cell therapy as a potential treatment for T1D to replace lost $\beta$ cells and to slow disease progression. Ultimately, stem cells would be a tool that health care professionals could use to avoid allogeneic transplantation and provide a dependable means for glucose control. The current landscape of stem cell biology, particularly in the context of T1D, is not completely clear; this review provides a detailed overview of each of the proposed and studied stem cell therapies and provides an evidence-based evaluation and comparison of their clinical merits.

\section{Stem Cell Therapies}

Stem cells are unspecialized cells that are capable of selfrenewal and can become specialized through the process of differentiation. Stem cells can be totipotent, pluripotent, or multipotent, which describes their range of cellular fates. Totipotent stem cells are capable of becoming all tissues in the body, including extraembryonic structures like the placenta, whereas pluripotent stem cells can become 3 embryonic germ layers (i.e., endoderm, ectoderm, and mesoderm). Induced pluripotent stem cells (iPSCs) and embryonic stem cells (ESCs) are the 2 major types of pluripotent stem cells. Multipotent cells are typically limited to becoming 1 germ layer and follow lineage-specific differentiation. Although many multipotent stem cells have been examined as a means to treat T1D, there are 2 types derived from the mesoderm that are actively being investigated: mesenchymal stem cells (MSCs) and hematopoietic stem cells (HSCs).

The 2 major goals of stem cell therapies in T1D include (1) replacing the loss of $\beta$-cell function and (2) regulating the immune system. Stem cells have significant therapeutic potential owing to their intrinsic regenerative capacity, allowing them to compensate for lost $\beta$-cell mass. To be bona fide $\beta$ cells, the following 2 criteria must be met: (1) have the ability to synthesize, store, and release mature insulin and (2) secrete an appropriate amount of insulin in response to ambient glycemia through glucose sensing. When differentiated cells meet the first criterion, they are referred to as insulin-producing cells (IPCs). The second criterion is usually achieved with varying degrees of success across protocols. The amount of insulin produced is often used to evaluate the quality (or similarity to $\beta$ cells). Cells that are capable of glucose sensing (i.e., release insulin in response to glucose stimulation) in vitro are referred to as $\beta$-like. IPCs (and $\beta$-like cells) are characterized by their protein products (e.g., insulin; C-peptide; and gene expression, e.g., $P d x 1$ and Nkx6-1) (4) and histological structures. ${ }^{5}$ These characteristics are monitored in derived cells to stage differentiation.

The second goal is to mitigate the effects of the immune system to preserve extant and transplanted tissue, facilitate endogenous $\beta$ cell regeneration from existing progenitor cells, and prevent autoimmunogenicity. Recent studies have focused on the 2 distinct methods of immunomodulation and encapsulation. Immunomodulation is broadly achieved by ablating or mitigating the effects of autoreactive $\mathrm{T}$ cells, promoting new regulatory $\mathrm{T}$ cells, altering proapoptotic signaling found in T1D, and improving the inherent immune tolerance of the body to $\beta$ cells. ${ }^{1}$ Eliminating immunosuppression would be ideal in terms of improving patients' health and quality of life. A possible solution to this problem is the recently developed method of encapsulation, which involves the physical separation of cells from the immune system. ${ }^{6,7}$

To achieve the 2 aforementioned goals, the many pros and cons of each therapy must be weighed. Biological characteristics, such as the ability to create new replacement IPCs or $\beta$ cells and modulate the immune system, teratogenicity, and immunogenicity, must be paired with practical questions of ethics, accessibility, scope, and efficacy to determine the future viability of a therapy.

\subsection{Embryonic Stem Cells}

ESCs are pluripotent stem cells that can become any cell in the body and may be used to replace lost $\beta$ cells. ESCs are harvested from the inner cell mass of blastocysts and can be grown and expanded in a dish. ESCs show exquisite plasticity in their 
undifferentiated state and are characterized by high levels of expression of Oct4, Nanog-1, and Sox 2 . $^{1}$

Pluripotent stem cells are most commonly differentiated into IPCs using a definitive endoderm (DE) intermediate that can become many endodermal cell types. ${ }^{4}$ Laboratory differentiation of cells is achieved by sequential exposure of factors (e.g., growth factors, transcription factors, and extracellular matrix proteins) under specific incubation regimens and can be measured by gene products, cytochemistry, and morphology. DE can be further differentiated in vitro to become pancreatic progenitor cells. Once grafted to the pancreas, the microenvironment enables the pancreatic progenitor cells to continue differentiating in vivo to become IPCs (i.e., make insulin, C-peptide, PDX1, and NKX6.1). ${ }^{4}$ Although this is addressed further on in this review, it is worth noting here that pancreatic progenitor cells can be directly differentiated into IPCs in vitro (5). The in vivodifferentiated IPCs made by Kroon et al. (2008) were assessed for their glucose-sensing capabilities; the IPCs responded appropriately to hypoglycemia and could rescue Streptozotocin (STZ)-induced diabetic mice (a chemical with a preferential toxicity towards pancreatic $\beta$ cells) from hyperglycemia, exhibiting $\beta$-like properties once grafted. ${ }^{4}$ An overview of this experiment (and those discussed later) is depicted in Figure 1. Additional studies examining whether IPCs made from stem cells had adverse effects found that they produce teratomas ${ }^{8}$ and need immunosuppression. ${ }^{1}$

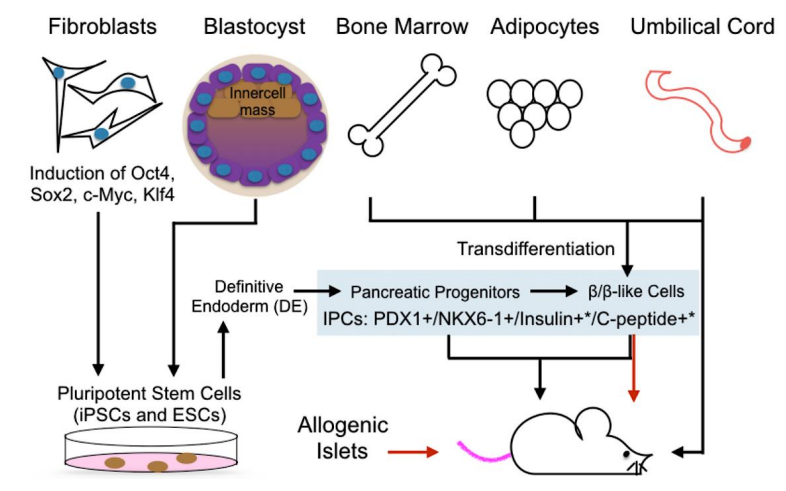

Figure 1. An overview of insulin-producing cell (IPC) experiments. The pluripotent and mesenchymal stem cell (trans)differentiation techniques and encapsulation experiments discussed in this review are highlighted in this flow chart. The cell types (pancreatic progenitors and $\beta / \beta$-like cells) in the blue box are both IPCs, although pancreatic progenitors do not produce insulin until they are put in vivo. The red arrows indicate experiments wherein encapsulation methods were evaluated. Note: The asterisk indicates pancreatic progenitor cells only express insulin and C-peptide after microenvironment differentiation once placed in vivo. ESCs indicate embryonic stem cells; iPSCs, induced pluripotent stem cells.

Two encapsulation methods have been developed to mitigate the negative impacts of cancer formation and immunosuppression of ESCs. Encapsulation adopts the principle of immunoisolation, which uses a physical, porous barrier that can selectively filter access to transplanted cells and protect them from the immune system. This barrier permits relatively small molecules, such as glucose, insulin, and electrolytes, to pass through the pores, whereas immune cells and antibodies would be too large. ${ }^{7}$ The first implementation of this innovative technology is the VC-01 produced by ViaCyte and is currently in clinical trials. This device is implanted subcutaneously, which makes it retrievable, and is immune-protecting. The form factor allows macroencapsulation of ESC-derived IPCs. Agulnick et al. (2015) showed that in vitro-differentiated IPCs in the VC-01 could increase levels of C-peptide in STZ-induced diabetic, immunecompetent mice in response to intraperitoneal glucose administration. ${ }^{6}$

The second method of encapsulation uses a newly identified, chemically modified triazole- thiomorpholine dioxide- (TMTD) alginate that resists implant fibrosis observed in both rodents and nonhuman primates. ${ }^{9}$ The TMTD-alginate has many of the same properties as the macroencapsulation (Figure 2). Vegas et al. (2016) showed that TMTD-alginate-encapsulated ESC-derived $\beta$ cells injected intraperitoneally into STZ-induced diabetic, immunecompetent mice could achieve glucose responsiveness and long-term glycemic control (Figure 1). ${ }^{7}$ The mice exhibited euglycemia for 174 days, and this may have lasted longer but the grafts were removed to end the experiment. In summary, these encapsulation methods have strong potential in future stem cell therapies.

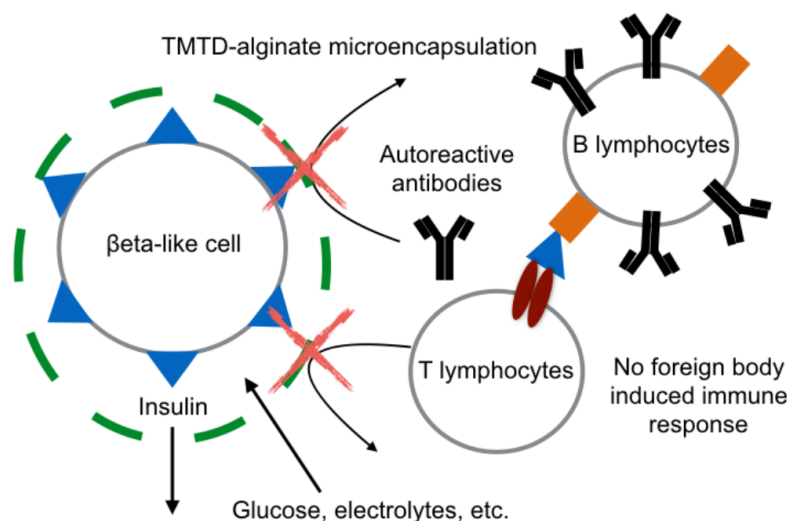

Figure 2. Triazole-thiomorpholine dioxide (TMTD)-algina microencapsulation. The [beta]-like cell is microencapsulated TMTD-alginate and protected from both the innate and autoimmur response-associated typoe 1 diabetes. The TMTD-alginate permi insulin, glucose and nutrients to freely pass.

\subsection{Induced Pluripotent Stem Cells}

Takahashi and Yamanaka (2006) first made iPSCs from mouse fibroblasts using retroviral transduction of transcription factors Oct4, Sox2, $c-M y c$, and Klf4. As pluripotent stem cells, iPSCs are also capable of becoming IPCs through a DE intermediate and have similar characteristics as ESCs. ${ }^{10,5}$ Many of the same differentiation protocols and experiments (e.g., rescuing STZ-induced diabetic mice with IPCs) (Figure 1) have been performed using iPSCs from fibroblasts. ${ }^{5}$

The benefits of iPSCs over ESCs are the use of autologous grafts, wherein the recipient is also the donor, obviating the ethical and moral controversy of using embryonic tissue, and increased accessibility. Maehr et al. (2009) provided an excellent proof-ofconcept by taking skin fibroblasts from patients with T1D and turning them into iPSCs and subsequently IPC $\beta$-like cells by in vitro differentiation. ${ }^{10}$ An autologous graft of IPCs generated this way could also help reduce the burden of immunosuppression. To our knowledge, there are no clinical trials using iPSCs to treat T1D, which could be largely owing to the tumorigenicity of iPSCs and to the fact they were developed after ESCs. The most common technique to make iPSCs involves viral insertions of oncogenes. There have been several advances in generating iPSCs without viral vectors using transient expression of the transcription factors on plasmids and microRNA (i.e., miR-302); ${ }^{1}$ however, most iPSC research in the literature uses the more established viral integration strategy. One study found that IPCs made by retrovirally transduced iPSCs make teratomas faster and more efficiently in all sites of injection compared with $\mathrm{ESCs},{ }^{8}$ creating controversy in the therapeutic potential of ESCs compared with iPSCs.

ESCs and iPSCs share many of the same pros and cons; however, iPSCs may have lower immunogenicity and higher tumorigenicity. There are also fewer ethical considerations and dilemmas in creating iPSCs compared with ESCs. If the tumorigenicity can be sufficiently mitigated using new techniques, iPSCs have a much better outlook for future use from both a scientific and ethical standpoint.

\subsection{Adipose Derived Stem Cells}

Adipose-derived stem cells (ADSCs) are MSCs and, as such, they have a hypoimmunogenic phenotype, lack MHC class II expression, instigate $P D-1$ suppression of $\mathrm{B}$ and $\mathrm{T}$ cells, release antiinflammatory cytokines, and can be turned into IPCs (1). Specifically, ADSCs are candidates for stem cell therapy because of 
their abundance in the human body and the relatively safe isolation protocol. ADSCs have the capability to differentiate into multiple cell lineages that can be engineered for stem cell-based treatment. These cells have been studied in diabetic animal models and in human clinical trials to help advance the current treatment of T1D. ${ }^{11}$ This study intravenously administered autologous undifferentiated ADSCs in STZ-induced Sprague-Dawley rats and resulted in reduced fasting glucose levels, increased expression of insulin, and improved islet injury over the span of 4 months (Figure 1). ${ }^{11}$ Such transplanted IPC clusters survived for 50 days after xenograft, producing insulin and C-peptide. These cells did not require immunosuppressive drugs after implantation owing to the lack of human leukocyte antigen surface protein expression. ${ }^{11}$ Transplanted ADSCs expel a variety of repair molecules to add to their therapeutic potential of the cell, including neurotrophic, immunomodulatory, and antioxidant factors. ADSCs are ideal for evading ethical and tumorigenic complications commonly found with the use of stem cell transplantation therapy. ${ }^{11}$ Because ADSCs can both create IPCs and immunomodulate, and because they lack any safety issues regarding genetic or epigenetic instability compared with other types of MSCs, they are an exciting, relatively well-understood potential therapy to be studied for T1D

\subsection{Bone Marrow Stem Cells}

Bone marrow stem cells (BM-MSCs) are a type of MSC that can be transdifferentiated in vitro into glucose-sensing IPCs and have the ability to promote endogenous $\beta$-cell regeneration through immunomodulation. BM-MSCs are harvested by aspirating BM and are then filtered to remove any unwanted material. ${ }^{1}$ In vitro studies have confirmed the immunomodulatory effects of BM-MSCs, successfully suppressing the activation of the body's immune response to antigens. One study conducted by Augello et al. (2005) demonstrated the ability of BM-MSCs to inhibit the activation and proliferation of both $\mathrm{T}$ and $\mathrm{B}$ lymphocytes through apoptosis by the PD-1 pathway. ${ }^{24}$ BM-MSCs are uniquely able to traffic specifically to islet cells, affecting their local environment to better induce their immunomodulatory effects. ${ }^{1}$ Hess et al. (2003) were the first to show immunomodulation of BM-MSCs when they stimulated endogenous pancreatic regeneration in mice after transplantation. ${ }^{25}$ A follow-up study by Lee et al. (2006) showed that transplanted human BMMSCs helped lower blood glucose levels in diabetic immunodeficient mice by facilitating the repair of pancreatic islets. ${ }^{26}$

Although BM-MSCs have the demonstrated immunomodulatory component of an ideal stem cell therapy regimen, the success of cell differentiation to IPCs has not been consistently reproducible. This is exemplified in a study by Choi et al. (2003), which demonstrated an inconsistency in the transdifferentiation of BM-MSCs into IPCs in an attempt to treat mice models with pancreatic injury (Figure 1). Currently, to our knowledge, there are no completed clinical trials using BM-MSCs to create IPCs. Therefore, at this time, BM-MSCs are not a viable option in attempts to derive future T1D stem cell therapies.

\subsection{Umbilical Cord Tissue and Blood}

Human umbilical cord tissue and blood are both sources of MSCs with the ability to differentiate into IPCs. Wharton jelly, a gelatinous undifferentiated tissue found in the umbilical cord, is rich in stem cells. Human umbilical cord Wharton jelly-derived MSCs (hUCMSCs) are adult stem cells capable of differentiating into several cell phenotypes. ${ }^{12}$ Not only are hUCMSCs easy to obtain, but the cells also display the unique self-renewal, plastic adherence, large differentiation potential in vivo, and immunomodulatory properties that prevent tissue rejection. ${ }^{12}$ A study conducted by Chao et al. (2008) successfully originated islet-like clusters derived from MSCs from the Wharton jelly of the human umbilical cord for transplantation to control T1D. Unfortunately, hUCMSCs have the tremendous practical burden of banking cord tissue, and the momentum to develop hUCMSC therapies has diminished because many current patients with T1D do not have the required stored tissue. $^{12}$

\subsection{Hematopoietic Stem Cells}

HSCs are the most clinically studied stem cell derivatives in the context of T1D. HSCs have the ability to self-renew and differentiate into cells of the myeloid and lymphoid lineages, and they provide the body with tremendous regenerative potential for these cell types (Figure 3). Considering the immunomodulatory effects of HSCs, researchers have long been reviewing the role HSCs may play in treating autoimmune diseases. ${ }^{1}$

Owing to the ease of the procedure, BM-derived HSCs (BMHSCs) have become the most commonly harvested cell type. This is primarily done by 2 methods: (1) direct aspiration of the BM under anesthesia or (2) collection of mobilized HSCs from circulating blood (Figure 3). In the latter technique, HSCs are mobilized into peripheral blood by the infusion of a chemotherapeutic agent, cyclophosphamide, and a growth factor, granulocyte colonystimulating factor (G-CSF). Cells are then isolated and collected using apheresis and then stored. ${ }^{13}$ These cells are then proliferated and positively and negatively selected. Specifically, HSCs are characterized as CD34 and Thy-1 positive and CD-38, CD-33, and HLA-R negative. To reduce an immunogenic response at transfusion or transplantation, the patient either undergoes extreme immunosuppression or a distinct type of chemotherapy known as myeloablation. ${ }^{1}$ Autologous and allogeneic therapies have both traditionally been used. It has been demonstrated that autologous therapy is more successful than allogeneic treatment. ${ }^{14}$

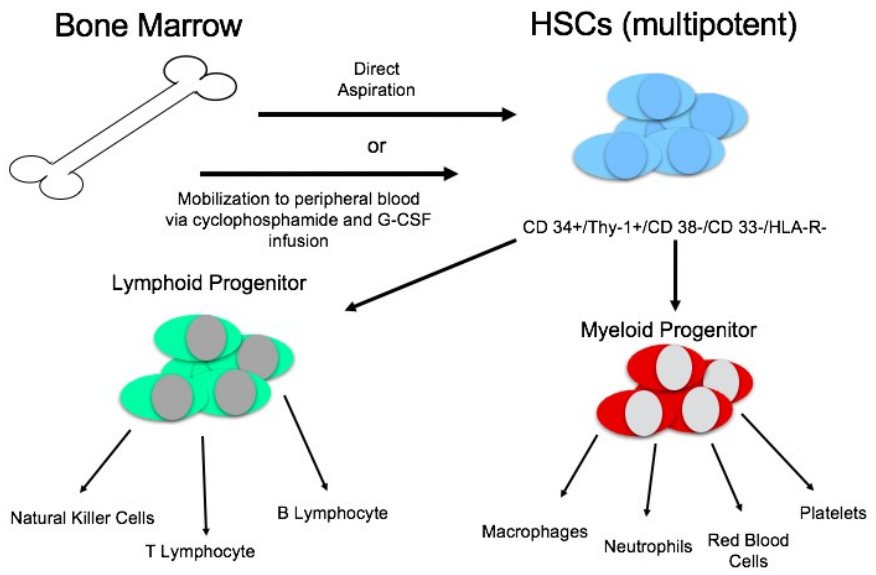

Figure 3. Hematopoietic Stem Cell (HSC) extraction and differentiation pathway. Currently, HSCs are most commonly isolated from human bone marrow owing to accessibility and ease of procedures. HSCs are obtained via direct aspiration of the bone marrow while placing the patient under anesthesia, or through mobilization of HSCs to peripheral blood via cyclophosphamide and granulocyte colony-stimulating factor (G-CSF). Cells are then isolated and collected via apheresis. HSCs are traditionally characterized as CD34 and Thy-1 positive and CD38, CD33, and HLA-R negative. In vivo, HSCs are able to differentiate into lymphoid and myeloid progenitors.

Direct recreation of IPCs or $\beta$ cells is not the goal and has not yet been demonstrated compared with the proposed MSC, iPSC, and ESC therapies previously discussed. ${ }^{1}$ Instead, transplantation or infusion of BM-HSCs aims to reduce or alter the underlying autoimmune pathology to protect any remaining $\beta$ cells and facilitate regrowth leading to remission of T1D. ${ }^{13}$ Hasegawa et al. (2007) first demonstrated this in T1D-induced mice, and bone marrow transplantation was shown to improve pancreatic function and contribute to $\beta$-cell regeneration. In particular, they were able to make the crucial distinction that immunosuppression in addition to HSC therapy, not just immunosuppression alone, was leading to the significant increase in pancreatic function, demonstrating that the HSCs themselves were vital to the protection and possible regeneration of existing $\beta$ cells. ${ }^{15}$

Despite such strong results, the precise mechanism of action is still unknown. It has been suggested that HSCs reduce the 
proapoptotic signals, such as Bad, Bax, and FasL, within $\beta$ cells Such signals are commonly found elevated in T1D. This mechanism would imply that HSCs prevent apoptotic cell death of $\beta$ cells in T1D. ${ }^{16}$ In addition to partial formation of a new nonpathological immune system from the HSC myeloid and lymphoid derivatives (Figure 3), this finding could help mitigate the autoimmune pathology and lead to the protection of the functional, and possible regeneration of existing, $\beta$ cells. These protective effects would ultimately lead to the demonstrated restoration of endocrine pancreatic function. ${ }^{16}$ This therapy is considered very promising and has been clinically studied.

\section{Clinical Comparison of Therapies and Recommendations}

Stem cell therapies for T1D are novel; divergent approaches and a relative lack of demonstrated clinical efficacy has led to an unclear landscape. There are many ways to treat T1D with stem cells; this review has characterized each based on its ability to (1) replace lost $\beta$ cell function and (2) regulate the immune system to combat or circumvent the underlying autoimmunity of T1D. Taking into account the clinical/biological criteria as well as the practical applications of the therapies, such as accessibility, scope, and ethics, this review provides insight into the therapies that show the most promise and thus deserve focus moving forward.

The innate biology of MSCs allowing for differentiation into IPCs, as well as the ability to modulate the immune system through hypoimmunogenic traits and regulation of $\mathrm{T}$ and $\mathrm{B}$ lymphocytes, makes these cells interesting potential therapies. ${ }^{1}$ However, MSCs derived from both human umbilical cords and BM have had mixed success in demonstrating such traits in practice. For BM-MSCs, the ability to improve T1D symptoms through immunomodulation has been experimentally and clinically demonstrated through the work of Carlsson et al. (2015). ${ }^{28}$ Twenty patients with new-onset T1D were given an infusion of BM-MSCs, and those with the infusion stabilized serum C-peptide compared with the control group. However, BM-MSCs have not been shown to faithfully differentiate into IPCs with high efficiency and will likely not see future applications. ${ }^{17}$ For hUCMSCs, there has been a general lack of clinical success as well. Haller et al. (2009) reported a negative result when using hUCMSCs, and patients did not show significant improvement in C-peptide production, insulin use, and any increase in regulatory T-cell levels. ${ }^{18}$ In addition to the practical concerns of banking, this result means that hUCMSCs will probably be pursued with limited interest. ${ }^{18}$ Taken together, evidence of experimental success and clinical efficacy for BM-MSCs and hUCMSCs to both modulate the immune system and differentiate into IPCs is not currently available and thus does not show strong clinical promise.

Contrary to MSCs derived from the umbilical cord and BM, ADSCs have repeatedly been shown to efficiently differentiate into IPCs, present favorable immunomodulatory traits associated with MSCs, and be easily obtained. ${ }^{11,19}$ Thus, ADSCs have been the focus of multiple successful clinical studies. The studies by Thakkar et al. (2015) and Trivedi et al. (2008) have indicated the efficacy and safety of a combined stem cell therapy using ADSCs and HSCs. ${ }^{14,29}$ Such outcomes included increased serum C-peptide levels, decreased incidence of diabetic ketoacidosis, and decreased insulin dependence. While the success of these therapies is not completely understood, it is likely explained by the actions from both lymphocyte cell types. ${ }^{19}$

HSCs have been the most studied cell type in the context of T1D treatment. Multiple clinical trials have demonstrated their efficacy and safety. ${ }^{20,13,21}$ A meta-analysis by El-Badawy and El-Badri (2016) pooled data from many of these recent trials to discern the progress of the clinical impact of treating T1D with HSCs. A total of 149 patients across 6 studies were followed up. HSCs were isolated using the procedure previously discussed, and 146 of the patients received nonmyeloablative, intravenous infusion of autologous HSCs. The remaining 3 patients received the cells via liver puncture. Overall, for those patients where C-peptide and hemoglobin A1C levels were recorded, the analysis demonstrated significant increases in serum $\mathrm{C}$ - peptide levels and a decrease in hemoglobin A1C levels after infusion. In addition, $58.6 \%$ of the patients receiving intravenous infusion $(n=146)$ demonstrated some degree of insulin independence. They were insulin free for an average of 16 months. ${ }^{22}$

Considering nonmyeloablative, autologous HSC infusion is the strongest clinically studied therapy to reverse or treat $\mathrm{T} 1 \mathrm{D}$, the demonstrated efficacy, as well as the reduction in oncogenicity compared with other stem cell lines, makes a strong case for continued interest in HSC therapy. ${ }^{1}$ However, there are some very important issues associated with HSC treatment. First, the current HSC therapy is more effective in patients earlier in their disease progression and in those who have not experienced diabetic ketoacidosis. ${ }^{22}$ Second, the immunosuppression component of the treatment is not ideal, as HSCs are immunogenic. Even in nonmyeloablative therapies, patients still undergo major immunosuppression and are left vulnerable to secondary infection. In particular, Daddio et al. (2014) observed adverse events in $52.3 \%$ of patients $(n=64)$, where 1 patient died of sepsis. ${ }^{13}$ Therefore, it is possible that the introduction of ADSCs with HSCs, as a combination therapy, may compensate for the insufficiencies of HSCs alone.

The introduction of IPCs may mitigate the importance of preventing disease progression in regard to treatment. With a combination therapy, the lost $\beta$ cells are directly replaced and not simply protecting any remaining endogenous functioning cells. Also, incorporating the additional immunomodulatory characteristics of ADSCs may further the regulation of autoreactive lymphocytes. This may continually improve the immune microenvironment in the pancreas to promote cell growth, as well as possibly reduce the need for strong immunosuppression, and thereby reducing the risk of secondary infections. ${ }^{19}$

Despite the possible benefits of this therapy, it is still relatively new, and much larger and powerful studies are needed to fully understand the treatment as a whole in terms of efficacy, safety, and scope for implementation as a standard of care. Even if this is achieved, this joint therapy is still not perfect. Patients further in their disease progression may still have a less favorable prognosis with the joint therapy. Also, a degree of immunosuppression will likely always be needed, directly influencing patient health and quality of life. Furthermore, the multipotency of the cells limit their differentiating ability. Although this therapy is plausible and likely efficacious in the treatment of T1D, it is not ideal. ${ }^{22,19}$

In viewing all aspects of the therapies discussed here, complications of immunocompromization in patients are a serious limitation. Traditionally, this is also a major limitation of therapies involving pluripotent stem cells when directly grafted. This is why immunoisolation via encapsulation is an attractive alternative, as it makes full use of the IPC-producing potential of ESCs and iPSCs by possibly limiting immune-related pitfalls. This technique is proposed to reduce the likelihood of cell death from underlying autoimmunity and mitigate the immunogenic traits of both pluripotent cells.

Early attempts at immunoisolation in patients showed that alginate-microencapsulated pancreatic islet allografts could achieve glycemic correction for short periods ( $>15$ days) in immunecompetent patients with T1D. ${ }^{23}$ They determined the short duration was due to the implants eliciting strong innate immune-mediated foreign body responses that resulted in fibrotic deposition on the alginate microsphere. The deposition caused the transplanted tissue to be isolated from nutrients and resulted in donor tissue necrosis with subsequent loss of blood glucose control. This study alluded to the potential use of TMTD-alginate, which Vegas et al. (2016) have since shown to resist fibrotic deposition in both rodents and nonhuman primates. ${ }^{7}$ Combining the TMTD-alginate encapsulation with pluripotent stem cell-derived IPCs could revolutionize stem cell therapy in the future.

The subcutaneous implantable device, VC-01, is the more mature form of encapsulation. The safety and efficacy of VC-01 for treating patients with T1D is currently in a phase 2 clinical trial (ClinicalTrials.gov identifier: NCT02239354). To our knowledge, this is the first clinical trial to test ESC-derived $\beta$-like cells, and the 
results will shape the outlook of their future use. Future clinical trials using both macro- and microencapsulation will be helpful in determining the leading choice for therapy. The greatest benefit of this treatment may be for patients suffering from advanced stages of T1D, where immunomodulation alone would not sufficiently restore glucose control. In summary, we have found that encapsulation of pluripotent stem cells differentiated into $\beta$-like IPCs have the most clinical promise in the treatment of T1D.

\section{Conclusion}

In our evaluation of current stem cell therapies for treating T1D, we found that there are 2 viable treatments with strong potential over current standards of care. These treatments fulfill both goals of T1D stem cell therapy by replacing the loss of $\beta$-cell function and regulating the immune system. The first is a combination therapy of nonmyeloablative, intravenous infusion of HSCs and ADSC-derived IPCs. This therapy elicits the HSCs' ability to protect and potentiate the possible regeneration of existing $\beta$ cells along with supplementation by ADSC IPCs. This proved to be a sufficient but not an ideal method to treat T1D owing to the concurrent need for immunosuppression. The uncertainty in the mechanism of action is a cause for concern. This may also be a more beneficial therapy for patients newly diagnosed as having T1D because there is still enough pancreatic tissue remaining that endogenous regeneration and immunoprotection can still be achieved.

The preferred choice for future stem cell therapy is likely to be encapsulation. This method uses in vitro differentiation of pluripotent stem cells into $\beta$-like IPCs and isolates them from the immune system. This not only prevents autoimmunogenicity to $\beta$ cells in T1D and other complications in graft versus host disease, but also protects against the adverse oncologic properties inherent to the use of stem cells. Owing to the biological similarities and ethical controversies between ESCs and iPSCs, we suggest that iPSCs should be used as the donor tissue source. We did not find that any other sources of $\beta$-cell induction (i.e., mesoderm-derived progenitors) could produce IPCs with the same efficiency as pluripotent stem cells. There are 2 competing methods of encapsulation being investigated: macro- and microencapsulation. There is currently a clinical trial underway using the $\mathrm{VC}-01$ macroencapsulation of ESCderived $\beta$-like IPCs, which we believe may benefit from the use of iPSCs. If it is shown that this subcutaneous implant can safely maintain blood glucose in patients with T1D, it will likely be widely used because it can be retrieved when maintenance is required. The TMTD-alginate microspheres used by Vegas et al. (2016) are not yet in clinical trials, but they have been successful in mouse models. ${ }^{7}$ The lack of sufficient clinical evidence necessary for a head-to-head comparison of encapsulation means that the definitive treatment remains elusive at the present time.

Currently, there is no cure for T1D, but recent advances in stem cell therapies have offered several new treatment options. We have found the most promising stem cell therapy to be one that combines in vitro differentiation of human stem cells into functional pancreatic $\beta$-like IPCs with encapsulation. The benefit of using stem cells to generate IPCs is that they provide an unlimited supply of tissue and can be encapsulated to eliminate the need for life-long immunosuppression.

\section{References}

1. Fiorina P, Voltarelli J, Zavazava N. Immunological Applications of Stem Cells in Type 1 Diabetes. Endocrine Reviews. 2011;32(6):725-754. doi:10.1210/er.2011-0008.

2. Bellin MD, Barton FB, Heitman A, et al. Potent Induction Immunotherapy Promotes Long-Term Insulin Independence After Islet Transplantation in Type 1 Diabetes. American Journal of Transplantation. 2012;12(6):1576-1583. doi:10.1111/j.1600-6143.2011.03977.x.
3. Krieger NR, Odorico JS, Heisey DM, et al. Underutilization of pancreas donors. Transplantation. 2003;75(8):1271-1276. doi:10.1097/01.tp.0000061603.95572.bf.

4. Kroon E, Martinson LA, Kadoya K, et al. Pancreatic endoderm derived from human embryonic stem cells generates glucoseresponsive insulin-secreting cells in vivo. Nat Biotech. 2008;26(4):443-452. doi:10.1038/nbt1393.

5. Pagliuca FW, Millman JR, Gurtler M, et al. Generation of functional human pancreatic beta cells in vitro. Cell. 2014;159(2):428-439. doi:10.1016/j.cell.2014.09.040.

6. Agulnick AD, Ambruzs DM, Moorman MA, et al. InsulinProducing Endocrine Cells Differentiated In Vitro From Human Embryonic Stem Cells Function in Macroencapsulation Devices In Vivo. Stem Cells Translational Medicine. 2015;4(10):12141222. doi:10.5966/sctm.2015-0079.

7. Vegas AJ, Veiseh O, Gurtler M, et al. Long-term glycemic control using polymer-encapsulated human stem cell-derived beta cells in immune-competent mice. Nat Med. 2016;22(3):306-311. doi:10.1038/nm.4030

8. Gutierrez-Aranda I, Ramos-Mejia V, Bueno C, et al. Human Induced Pluripotent Stem Cells Develop Teratoma More Efficiently and Faster Than Human Embryonic Stem Cells Regardless the Site of Injection. Stem Cells. 2010;28(9):15681570. doi:10.1002/stem.471.

9. Calafiore R. Microencapsulated Pancreatic Islet Allografts Into Nonimmunosuppressed Patients With Type 1 Diabetes: First two cases. Diabetes Care. 2006;29(1):137-138. doi:10.2337/diacare.29.1.137.

10. Maehr R, Chen S, Snitow M, et al. Generation of pluripotent stem cells from patients with type 1 diabetes. Proc Natl Acad Sci USA. 2009;106(37):15768-15773. doi:10.1073/pnas.0906894106.

11. Lin H-P, Chan T-M, Fu R-H, et al. Applicability of AdiposeDerived Stem Cells in Type 1 Diabetes Mellitus. Cell Transplantation. 2015;24(3):521-532. doi:10.3727/096368915x686977.

12. El-Hossary N, Hassanein H, El-Ghareeb AW, Issa H. Intravenous vs intraperitoneal transplantation of umbilical cord mesenchymal stem cells from Wharton's jelly in the treatment of streptozotocin-induced diabetic rats. Diabetes Research and Clinical Practice. 2016;121:102-111. doi:10.1016/j.diabres.2016.09.008.

13. Daddio F, Vasquez AV, Nasr MB, et al. Autologous Nonmyeloablative Hematopoietic Stem Cell Transplantation in New-Onset Type 1 Diabetes: A Multicenter Analysis. Diabetes. 2014;63(9):3041-3046. doi:10.2337/db14-0295.

14. Thakkar UG, Trivedi HL, Vanikar AV, Dave SD. Insulinsecreting adipose-derived mesenchymal stromal cells with bone marrow-derived hematopoietic stem cells from autologous and allogenic sources for type 1 diabetes mellitus. Cytotherapy. 2015;17(7):940-947. doi:10.1016/j.jcyt.2015.03.608.

15. Hasegawa Y, Ogihara T, Yamada T, et al. Bone Marrow (BM) Transplantation Promotes $\beta$-Cell Regeneration after Acute Injury through BM Cell Mobilization. Endocrinology. 2007;148(5):2006-2015. doi:10.1210/en.2006-1351.

16. Oliveira GLVD, Malmegrim KCR, Ferreira AF, et al. Upregulation of fas and fasL pro-apoptotic genes expression in type 1 diabetes patients after autologous haematopoietic stem cell transplantation. Clinical \& Experimental Immunology. 2012;168(3):291-302. doi:10.1111/j.1365-2249.2012.04583.x.

17. Choi JB, Uchino H, Azuma K, et al. Little evidence of transdifferentiation of bone marrow-derived cells into pancreatic beta cells. Diabetologia. 2003;46(10):1366-1374. doi:10.1007/s00125-003-1182-9.

18. Haller MJ, Wasserfall CH, Mcgrail KM, et al. Autologous Umbilical Cord Blood Transfusion in Very Young Children With Type 1 Diabetes. Diabetes Care. 2009;32(11):2041-2046. doi:10.2337/dc09-0967. 
19. Vanikar AV, Trivedi HL, Thakkar UG. Stem cell therapy emerging as the key player in treating type 1 diabetes mellitus. Cytotherapy. 2016;18(9):1077-1086. doi:10.1016/j.jcyt.2016.06.006.

20. Chen L, Dong J, Hou W, et al. Sustained Effect Of Autologous Bone Marrow Mononuclear Cell Transplantation In Patients With Diabetes 12 Month Follow-Up. Transplantation. 2008;86(Supplement): 162 . doi:10.1097/01.tp.0000332277.56852.b8.

21. Voltarelli JC, Couri CE, Stracieri AB, et al. Autologous nonmyeloablative hematopoietic stem cell transplantation in newly diagnosed type 1 diabetes mellitus. JAMA. 2007;297(14):1568-1576. doi:10.1001/jama.297.14.1568.

22. El-Badawy A, El-Badri N. Clinical Efficacy of Stem Cell Therapy for Diabetes Mellitus: A Meta-Analysis. PLoS One. 2016;11(4):e0151938. doi:10.1371/journal.pone.0151938.

23. Calafiore R. Microencapsulated Pancreatic Islet Allografts Into Nonimmunosuppressed Patients With Type 1 Diabetes: First two cases. Diabetes Care. 2006;29(1):137-138. doi:10.2337/diacare.29.1.137.

24. Augello A, Tasso R, Negrini S, et al. Bone marrow mesenchymal progenitor cells inhibit lymphocyte proliferation by activation of the programmed death 1 pathway. European Journal of Immunology. 2005;35(5):1482-1490. doi:10.1002/eji.200425405.

25. Hess D, Li L, Martin M, et al. Bone marrow-derived stem cells initiate pancreatic regeneration. Nature Biotechnology. 2003;21(7):763-770. doi:10.1038/nbt841.

26. Lee RH, Seo MJ, Reger RL, et al. Multipotent stromal cells from human marrow home to and promote repair of pancreatic islets and renal glomeruli in diabetic NOD/scid mice. Proc Natl Acad Sci USA. 2006;103(46):17438-17443. doi:10.1073/pnas.0608249103.

27. Chao KC, Chao KF, Fu YS, Liu SH. Islet-Like Clusters Derived from Mesenchymal Stem Cells in Whartons Jelly of the Human Umbilical Cord for Transplantation to Control Type 1 Diabetes. PLoS ONE. 2008;3(1). doi:10.1371/journal.pone.0001451.

28. Carlsson P-O, Schwarcz E, Korsgren O, Blanc KL. Preserved $\beta$ Cell Function in Type 1 Diabetes by Mesenchymal Stromal Cells. Diabetes. 2014;64(2):587-592 . doi:10.2337/db14-0656.

29. Trivedi H, Vanikar A, Thakker U, et al. Human Adipose TissueDerived Mesenchymal Stem Cells Combined With Hematopoietic Stem Cell Transplantation Synthesize Insulin. Transplantation Proceedings. 2008;40(4):1135-1139. doi:10.1016/j.transproceed 2008.03 .113 . 